\title{
A proposito di trobairitz
}

Marirì Martinengo

Liceu de Catanzaro

\section{Maestre di civiltà}

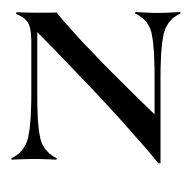

ella Provenza dei secoli XII e XIII c'è società femminile, dove per società femminile intendo un contesto sociale in cui viene tenuta in conto la parola delle donne; la storica spagnola Marìa Milagros Rivera Garretas dà della società femminile un'altra definizione, ${ }^{1}$ sulla quale anche concordo, e che si addice alla civiltà occitana: società in cui energie femminili sono dirottate prioritariamente a favore delle altre donne. Le trobairitz sono espressione di questa società. Un'altra storica, Régine Pernoud, afferma che "all'inizio del XIII secolo la storia fu fatta dalle donne". ${ }^{2}$ In questo periodo alcune donne esercitarono autorità in campo politico e culturale: Eleonora d'Aquitania, le sue figlie Maria di Champagne e Giovanna di Tolosa, le nipoti Bianca di Castiglia, Costanza e Isambour; né sono da dimenticare le feudatarie occitaniche come Adelaide di Burlat, Esclarmonde di Foix, Ermengarda di Narbonne, le signore del Monferrato e Malaspina in Italia che tenevano corte nei castelli da loro amministrati. Fra queste desidero ancora ricordare Gaia da camino che, divenuta sovrana, nel 1293, di Porto Buffolé, nel Trevigiano, tenne corte nella sua bella casa affrescata, dove, poeti e poetesse, profughi dalla terra d'oc, trovarono l'ambiente propizio per continuare a musicare e poetare, contribuendo alla formazione della lingua franco-veneta (Il Trevigiano è detto ancor oggi Marca Gioiosa per la folta presenza all'epoca di trovatori e trobairitz.

In queste corti le trobairitz alimentarono la cultura dell'epoca, senza dubbio influirono sul rapporto donna-uomo, donna-donna, che

\footnotetext{
${ }^{1}$ Marìa Milagros Rivera Garretas, cfr. Vias de bùsqueda de existencia femenina libre: Perpetua, Christine de Pizan, Teresa de Cartagena, in Duoda. Revista de estudis Feministes, sn. 5, 1993, p. 51.

${ }^{2}$ Régine Pernoud, Bianca di Castiglia, tr. it. P. Della Sala, ECIG, Genova, 1994, p.13.
} 
contribuirono a civilizzare. La Signora (che molto sovente è la dompna cantata dai trovatori e loro protettrice) si pose al centro della vita di corte; in questo centro le trobairitz fecero diventare l'amore, le relazioni interpersonali - basti osservare quanto siano frequenti le tenzoni, ${ }^{3} \mathrm{i}$ partimen (tenzoni di argomento estetico/etico), il dialogo, il confronto il nodo centrale, la questione fondamentale dell'esistenza, nella vita come nell'arte; è l'amore, interpretato da loro, che detta e regola il comportamento, cioè fonda l'estetica. In molte di queste poesie, in alcune canzoni, ma soprattutto nelle tenzoni, si manifesta l'estetica trobadorica femminile (uso di proposito il termine estetica e non quello di etica):

Penso che l'etica è nel regime di significato fatto di antinomie e di norme generali astratte ed ereditabili come da routine, senza partire da sé. Mi sembra invece che le Trovatore si svilupparono e si significarono in un'estetica, estetica intesa come arte e senso della percezione $($ dal greco aisthénesthai $=$ percepire $)$, sapienza estetica mai banale, che si fa e si disfa fra esseri umane e umani, singolari e vive, insegnando a godere della bellezza e ad armonizzare, per quanto possibile, a partire dalla relazione concreta di autorità, le contrarietà del desiderio e i paradossi della condizione umana storica. ${ }^{4}$

Nella tenzone dell' Anonima II due donne discutono tra loro di estetica amorosa:

Buona dama, tanto mi siete cara che non posso astenermi dal consigliarvi il vostro bene: e vi dico con sicurezza che commettete una grave scortesia, perché lasciate morire colui che mai amò

\footnotetext{
${ }^{3}$ La tenzone è un dialogo in poesia, in cui due interlocutrici o un'interlocutrice e un interlocutore, mettono a confronto i loro punti di vista su una questione; solitamente le due voci si alternano, strofa per strofa, ma non sempre viene rispettato questo schema; poiché la poesia trobadorica era accompagnata dalla musica, dal canto e, in una cornice fastosa, presupponeva un pubblico, si può dire che la tenzone inscenava un'azione drammatica.

${ }^{4}$ Maria Milagros Rivera Garretas esprime questo pensiero sia nella sua traduzione in spagnolo de Le Trovatore, poetesse dell'amor cortese (Las Trovadoras. Poetisas del amor cortés. Madrid Horas y horas, 1997, nota 9, p. 35) sia nella recensione dello stesso libro, in Duoda. Revista de estudios feministas, 12, 1997, p.163165; la traduzione è mia.
} 
alcuna cosa più di voi, e senza motivo; ma se muore sarà vostro il danno, ché nessun altra donna gli è tanto grata, né alcuna ha su di lui potere e signoria. Madamigella, ben devo essere crudele, quando si prende gioco o si vanta di me: possiede un cuore tanto folle e volubile che la mia amicizia non tiene in nessun conto, per cui il mio amore non gli interessa e non gli preme, e poiché è lui ad aver commesso una pazzia, non mi giudicate se vi vedo la follia, ché sento dire che è giusto ed onorevole.

Buona Dama, potete farlo bruciare o impiccare, o far tutto ciò di cui vi venga voglia, ché non vi è cosa ch'egli possa vietarvi...5

L'una, una donna sposata, ricorre alla parola di un'altra donna, di cui si fida, una damigella, sulla questione cruciale: può l'uomo incivile essere preso in considerazione dalla donna che ama? Nella seconda strofa c'è il ritratto di come non deve essere il cavaliere, mentre nella sesta c'è il ritratto del cavaliere meritevole d'amore:

Se vuole che gli renda il mio amore, Madamigella, ben è necessario che sia cortese e prode, sincero e umile, che con nessuno entri in contesa, e che sia amabile verso tutti;

che non mi piace un uomo malvagio e orgoglioso, a causa del quale il mio pregio decada o diminuisca, ma sincero e fedele, discreto e innamorato: se vuole che gli conceda mercé, che mi ascolti. $\ldots$

Nel congedo la damigella viene richiesta di farsi arbitra e garante di un rapporto amoroso equilibrato:

${ }^{5}$ Il testo in lingua d'oc di questa poesia, come di tutti i testi successivi, si trovano in Marirì Martinengo, Le Trovatore, poetesse dell'amor cortese, Milano, Libreria delle Donne, 1996 e in Marirì Martinengo, Le Trovatore, poetesse e poeti in conflitto, Milano, Libreria delle Donne, 2001. 
Madamigella, è lieto il finale, purché non deviamo, e voi siate guardiana fra noi, e prendete le parti di chi subisca torto.

Anche nella tenzone, che viene indicata dal primo verso, Alais Iselda e Carenza, due donne, apparentemente più giovani, chiedono ad un'altra, più anziana ed esperta, consigli su come regolarsi nella vita futura.

Donna Carenza, dal bel grazioso corpo, date consiglio a noi due sorelle e poiché sapete meglio scegliere la cosa migliore, consigliatemi...

Le trobairitz, espressione dell'autorità e della libertà che, insieme ad altre donne, si erano guadagnate, dicono a quali requisiti deve rispondere l'uomo affinché ottenga di potersi mettere in rapporto con loro, e più generalmente con le donne. ${ }^{6}$ La Contessa di Dia, una tra le poche largamente note delle trobairitz, dice:

\section{Una dama che miri al buon pregio ben deve porre il suo intento su un prode cavaliere valoroso dacché conosce il suo valore;}

La rappresentazione del rapporto uomo-donna presente nell'epica franca differisce profondamente dalla rappresentazione dello stesso che vediamo nella poesia cortese: nella Chanson de Roland, dell'XI secolo, Aude, la fidanzata del protagonista, cade a terra svenuta, quando egli le comunica la sua partenza per la guerra, ma Rolando si allontana senza voltarsi indietro; alla fine, dopo la morte di Rolando, Carlomagno, senza curarsi dei sentimenti di lei, le propone rudemente un nuovo fidanzato nella persona del proprio figlio; ma "ude, che Asa" di non poter sopravvivere dopo la morte dell'uomo amato, alla notizia della morte di Rolando, si affloscia a terra e muore. Che differenza con le donne occitane che, in autonomia, stabiliscono i termini del rapporto, e scelgono l'uomo

\footnotetext{
${ }^{6}$ A questo proposito ricordo anche la funzione civilizzatrice, in questo senso, delle giovani di Ratisbona, educande o novizie, vissute nel X secolo, che lasciarono testimonianza scritta del loro sentire e pensare; le trobairitz conobbero i loro versi?
} 
degno di essere amato! Così come antitetici appaiono, sempre nella stessa Chanson, i due cavalieri Rolando e Ulivieri, il primo esponente della dura e barbarica armata nordica, il cui eroismo sta nella forza e nella spada, il secondo paladino della civile e cortese cavalleria occitanica, il cui ardore è sempre temperato dalla mesura (equilibrio, moderazione).

\title{
Voce che parla ascoltata
}

Nella sua lunghissima canzone, propriamente un salut, Azalais d'Altier si rivolge ad un'altra trobairitz, Clara d'Anduza, dimostrandole affetto e grande considerazione; ricorre a lei per perorare in favore di un suo innamorato:

\author{
Molti saluti e molto amore \\ e tanto bene e tanto onore \\ e tanta cortese amicizia \\ e tanta gioia, quanta ne desiderate, \\ e tanti sorrisi e tanta allegria \\ vi manda Donna Azalais d'Altier; \\ a voi, signora, che vorrei \\ più d'ogni altra cosa al mondo vedere; \\ ché tanto bene ne ho udito parlare \\ da colui che è vostro servitore, \\ che per il bene che me ne ha detto, \\ ho talmente impressa nel mio cuore \\ la vostra immagine, che se vi vedessi, \\ fra mille vi riconoscerei. \\ $\mathrm{E}$ vi dico che in verità \\ non ho mai amato sinceramente \\ una donna, senza conoscerla;
}

Azalais si prodiga per l'armoniosa ricomposizione di un rapporto amoroso compromesso; la richiesta di mediazione proviene da un uomo famoso in ambito provenzale, Uc di Saint Circ - è infatti uno degli estensori delle vidas ${ }^{7}$ di trovatori e trobairitz - che si affida alla parola di una donna per riavere ciò che gli sta più a cuore:

${ }^{7}$ Le Vidas sono biografie un po' romanzate e non sempre attendibili storicamente. 
Ora, Signora, accade questo.

L'altro giorno venne presso di me

il vostro amico, triste e smarrito

come chi è accusato e tradito,

e mi disse che nelle parole e nei fatti,

secondo quello che voi diceste...

Anche qui l'autrice, nel congedo, si offre quale garante del felice proseguimento della sollecitata e poi effettivamente avvenuta (abbiamo la risposta grata di Uc) riconciliazione.

Ancora nella tenzone scritta da Bona Domna un trepido innamorato insiste nel chiedere consiglio ad una signora circa il modo di comportarsi con la donna che ama:

Buona Dama, vi chiedo un consiglio:

datemelo, poiché ne ho gran bisogno,

ché ho posto tutto il mio cuore in una donna,

e non cerco né desidero altrettanto alcuna cosa;

e ditemi se approvate che io chieda

la sua amicizia, o se ciò mi farà soffrire.

Ché il proverbio giustamente afferma:

perde chi ha fretta, e ottiene chi attende.

Signore, io vi dico, secondo il mio parere,

che agisce bene chi si dichiara a una donna cortese,

e che è poco saggio chi la teme;

che mai una dama ferì un cavaliere,

ma se non le piace che le dichiari il suo amore,

non vi nasce danno, in nessuna maniera, poiché una nobile dama ha tanta cortesia da sapersi negare con gentili parole.

Madonna, io temo che, se le domando il suo amore, mi risponda in malo modo,

e che si vanti del suo pregio e del suo onore,

e che mi dica che giammai mi amerà.

Sarebbe meglio, io credo, se continuassi a servirla,

finché le piaccia rendermi il guiderdone.

E ditemi, secondo il vostro sapere,

se farò bene o se mi sbaglio... 
Il finale della poesia è a sorpresa. Il fatto che queste richieste e così le risposte fossero espresse in linguaggio poetico sta a significare - tenendo conto che nella cerchia trobadorica la poesia accompagnata dalla musica era forma espressiva perfetta - la vitale importanza che si annetteva alle questioni.

Ruolo di educatrice, riconosciuto da Giraut di Borneil, assume Alamanda nella sua tenzone:

Se vi chiedo consiglio, bell'amica Alamanda, non me lo negate, ché ve lo domanda un uomo accasciato, poiché la vostra signora ingannatrice m'ha detto che molto ho deviato dal suo comando, che ciò che mi diede, ora mi toglie, e mi allontana; cosa mi consigliate? per poco il cuore non mi divampa d'ira, tanto sono afflitto.

In nome di Dio, Giraud, la volontà dell'amante non si compie affatto senza tener conto dell'altro; ché, se uno sbaglia, l'altro conviene faccia finta di nulla, in modo che il loro contrasto non cresca né s'espanda; e se ella vi ha detto che un'alta montagna è una pianura, voi dovete crederle, e vi piaccia il bene ed il male che vi manda: in questo modo sarete amato.

Infatti le trobairitz, maestre d'amore, assunsero su di sé il ruolo di educatrici - l'amore educa, è la filosofia cortese B e questo ruolo venne loro riconosciuto pienamente; esse affermavano di essere disposte ad amare solo un uomo cortese e così facendo contribuirono ad elevare, a civilizzare la società. Felipa raccomanda ad Arnaut Plagues:

E allora fa' quel che ti dico: mostrati elegante e cortese, gentile e di gradite maniere, e agisci secondo decoro, per quanto puoi e senza indugi, che così deve l'innamorato

In amore ci vuole mesura, così ci dice la Bona Domna nella sua tenzone con un trovatore di nome Pistoleta: 
Signore, sempre il folle corre verso la follia, ma è folle chi commette follia e quando un uomo serve là dove non è valore, poi se ne pente, poiché non ne ha alcun guadagno; anzi deve sapere di guardarsi dallo scoprirsi, se non può averne ricompensa o mercede;

In un partimen (tenzone che suggerisce il corretto comportamento fra l'innamorato e l'innamorata) che ha l'andamento di una disputatio accademica, assistiamo a un confronto serrato tra Maria di Ventadorn e Gui d'Ussel, nel quale la poetessa e il poeta espongono e dibattono, da punti di vista differenti, il codice del comportamento cortese e, in particolare, i rapporti tra la donna e l'uomo che si amano; le loro opinioni divergono:

Gui d'Ussel, mi lagno di voi, perché avete smesso di cantare, e dato che vorrei riportarvi alla poesia - in quanto siete molto saggio a tal riguardo voglio che mi diciate se la donna deve agire nei confronti dell'amante, quando lo ama sinceramente, allo stesso modo in cui l'amante agisce verso di lei in tutto quanto concerne l'amore, secondo la legge che vincola gli amanti.

Donna Maria, io volevo abbandonare tenzone e ogni altra forma di canto; ma ora non posso esimermi dal rispondere alla vostra domanda; e rispondo brevemente circa la dama: essa deve agire nei confronti dell'amante allo stesso modo in cui l'uomo agisce verso di lei, senza tener conto del grado nobiliare, ché, fra due amanti, non vi deve essere un superiore.

Gui, l'amante deve domandare per pietà tutto ciò di cui è desideroso, e la donna lo può comandare, e, a volte, deve supplicarla molto. L'amante deve rivolgere le sue preghiere e le richieste al contempo ad una dama e ad una amica 
mentre la dama deve onorare il proprio amante come un amico, non come un signore.

Ancora un confronto tra le posizioni di una donna e quelle di un uomo - tutta la poesia delle trobairitz riflette la consapevolezza che uomini e donne sono differenti, per cui percepiscono in modo differente - vediamo nel partimen di Gullelma di Rosers:

Donna Gullelma, molti cavalieri, girovagando

di notte, persa la strada per il maltempo, cercavano, lamentandosi nella loro lingua, un rifugio;

li udirono due che, per ragioni d'amore, se ne andavano in fretta verso le loro dame;

l'uno tornò indietro per aiutare quella gente, e l'altro si diresse correndo verso la propria dama; quale dei due fece meglio ciò che si conveniva?

Amico Lanfranco, meglio compì il suo viaggio, a mio parere, quello che proseguì verso l'amica;

l'altro fece bene, tuttavia la sua signora non può conoscere il suo nobile cuore altrettanto bene come quella che vide presente davanti ai suoi occhi il suo cavaliere di cui ha atteso l'arrivo; e vale molto più colui che fa ciò che ha promesso di colui che muta il suo proposito.

dove la donna parte da sé, dal proprio desiderio, mentre il trovatore, Lanfranco Cigala, obbedisce a norme di etica sociale. Tra i due dialoganti, che esprimono punti di vista differenti, c'è ascolto, rispetto, considerazione per le argomentazioni dell'altro, dell'altra. E questo vale per tutti i documenti e le testimonianze della civiltà cortese.

\section{Amore come via al melhorament}

Nel partimen, che stiamo analizzando, si vede quanto radicata fosse la consapevolezza dell'influenza dell'amore sui comportamenti sociali:

...tutto quanto fece di cortese

il cavaliere che grazie al suo coraggio

salvò gli altri dalla morte e dalle sofferenze, 
gli fu suggerito dall'amore, ché nessuno possiede affatto cortesia, se non gli proviene dall'amore.

Il trovatore, che preferì recarsi dalla donna amata prima di soccorrere i pellegrini stranieri in difficoltà, secondo Gullelma, aveva agito giustamente perché è nel dialogo amoroso con la donna la scaturigine del retto comportamento sociale (oltre che interpersonale): prima si va ad attingere sapere poi lo si mette in pratica:

perché non servì innanzi tutto la sua dama?

Ne avrebbe avuto grazie da lei e da loro stessi:

poi, per amor suo, avrebbe potuto servire

Molte degne persone e non avrebbe errato.

Nella tenzone dell'Anonima III le prime strofe sono poste in bocca a un uomo che constata come la mancanza di corrispondenza amorosa abbia effetto negativo sulla sua indole e sul suo comportamento; nell'ultima parte della poesia è la donna che parla:

Se il mio canto mi piacesse, mi sforzerei

e mi darei gioia, gaudio e piacere, ma sono portato a tal punto a scoraggiarmi poiché la mia dama, che ogni giorno disapprova ciò che le dico, non si degna di considerarlo: a malapena so rimanere fra i prodi e non sono affatto l'uomo che ero un tempo: così ella mi priva della mia cortesia e mi distrugge.

Ahimé ! come muoio quando mi sovviene come ero gaio e giovane, allegro, euforico! e quando mi accorgo che mi sono allontanato dalla gioia, per poco il mio cuore non si dispera del tutto; e dunque come i miei occhi potranno vederla, dal momento che ho perso il potere su essi e su me? Questo mi ha fatto, per cui il mio cuore va lamentandosi, perché non posso mostrare gentilezza né cortesia. $\cdots$

Amico dolce e bello, più non si lagnerebbe di me il vostro nobile cuore, cortese e saggio se conoscesse qual è il mio intento: voi siete colui per il quale oggi sono migliore di ieri; 
e non crediate che io vi tenga in poco conto, ché non posso trovare gioia perfetta senza di voi, a cui mi sono donata sinceramente, senza inganni, e vi lascio il mio cuore in pegno, ovunque vado.

"Voi siete colui per il quale oggi sono migliore di ieri", questo verso, che direi emblematico della cultura provenzale, esprime la fede nella potenza positiva dell'amore terreno. Nella civiltà provenzale dei secoli XII e XIII (civiltà distrutta nei primi decenni del XIII secolo dalle congiunte forze papali e franche durante la cosiddetta "crociata contro gli Albigesi" (12091243), gli ideali cortesi - raffinatezza, cultura, lealtà, fedeltà - non sono ritenuti appannaggio di poche pochi elette/i, ma si pensa debbano improntare l'intera società.

\section{Tutto ha origine nel valor e nel pretz, cioè nelle qualità della dama}

L'amore nel cuore del trovatore nasce sì dalla contemplazione della bellezza della signora, ma essenzialmente dalla considerazione delle sue doti intellettuali e spirituali; l'amore trae origine dall'ammirazione per l'aspetto esteriore, ma sono le affinità elettive a sollecitarlo. Nello stesso tempo il poeta ha coscienza della sua inferiorità nei confronti della dama e, per avvicinarsi a lei, si impegna in un melhorament che gradualmente otterrà, sviluppando le qualità che la donna richiede per corrispondergli. Dovrà raggiungere la mesura, cioè il controllo degli impulsi, degli istinti, delle ambizioni. In amore la mesura consiste nel provare alla signora che ella non è semplice oggetto di desiderio, e che l'amore è sentimento più che istinto. In tal modo il poeta sarà ammesso all'intimità della donna amata e ad essere spettatore di gesti e scene pervase di sottile erotismo e percorse da delicata sensualità.

L'amore trobadorico non si limita a dar forma al rapporto a due, ma intende permeare la società in modo che la convivencìa, cioè l'arte di vivere insieme nel rispetto della libertà reciproca, nella tolleranza, nella caritat diventi senso comune e la larguesa (la generosità) tanto morale quanto materiale diventi stile di vita. Cioè il culto dell'amore, con tutto quanto implica, eleva il tono dei rapporti interpersonali e sociali. 
Un filo sottile, ma continuo e tenace, percorre la nostra cultura occidentale: il riconoscimento della forza creatrice e civilizzatrice dell'amore di cui la donna è detentrice privilegiata se non esclusiva: da Diotima, maestra di Socrate, alla Venere altrice di Lucrezio, alla dompna della Terra d'oc, alla Signora Amore di Margherita Porete, alla Beatrice di Dante, alla Vergine Maria di San Bernardo e dei cistercensi; e si potrebbe continuareY ma io sono "rimasta" al mio Medioevo.

\section{La passione amorosa}

In altre poesie, che sono in forma di canzone, cioè dove la voce cantante è unica, le trobairitz esaltano la passione amorosa, anche se di rado c'è scissione tra l'erotismo e l'aspetto spirituale dell'amore.

La Contessa di Dia:

Sono stata in grave angoscia per un cavaliere che ho avuto, e voglio che per sempre sia risaputo come l'ho amato a dismisura; ora m'accorgo d'essermi ingannata, poiché non gli ho donato il mio amore, per cui ho vissuto nell'errore sia nel letto che vestita.

Come vorrei una sera tenere il mio cavaliere, nudo, tra le braccia, ch'egli si riterrebbe felice se solo gli facessi da guanciale; che ne sono più incantata di quanto Fiorio di Biancofiore; io gli dono il mio cuore e il mio amore, la mia ragione, i miei occhi e la mia vita. Bel amico, amabile e buono, quando vi avrò in mio potere? Potessi giacere al vostro fianco una sera, potessi darvi un bacio appassionato! Sappiate, avrei gran desiderio di avervi al posto del marito, con la condizione che mi concedeste di far tutto ciò ch'io volessi. 
La Contessa canta la gioia che dà l'amore, esalta la dama che ama senza nasconderlo:

Di gioia e gioventù m'appago, e gioia e gioventù m'appagano ché il mio amico è il più gaio, per cui sono graziosa e gaia: e poiché sono con lui sincera ben pretendo che sia con me sincero; che mai d'amarlo non mi astengo né ho cuore d'astenermene.

e ancora:

La gioia cortese mi dona felicità per cui canto più gaiamente e non mi dispiace per nulla né mi dà alcuna preoccupazione sapere che vogliono il mio danno falsi e vili lauzingier (maldicenti) e le loro parole malevole non mi spaventano: anzi ne sono due volte più gaia.

L'aube (alba) dell' Anonoma IV:

In un giardino, sotto le foglie di un biancospino, la donna tiene il suo amico vicino, finché la sentinella annuncia l'alba.

Oh Dio! Oh Dio! L'alba come viene presto!

Piacesse a Dio che la notte non finisse e che il mio amico da me non si partisse, e che la sentinella giorno od alba non vedesse.

Oh Dio! Oh Dio! L'alba come viene presto!

Amico dolce e bello, baciamoci io e voi, laggiù sui prati dove cantano gli uccelletti ... Amico dolce e bello, facciamo un nuovo gioco nel giardino dove cantano gli uccelli....

Tibors si esprime, nel frammento che di lei ci è rimasto, con dolci parole di desiderio e di nostalgia: 
Amico dolce e bello, ben posso dirvi in vero che mai accadde che io mancassi di desiderio dacché vi piacque che vi tenessi come cortese amante; né mai accadde, amico dolce e bello, ch'io non desiderassi vedervi spesso; né mai un momento me ne pentii; né mai accadde, se vi allontanavate irato, ch'io provassi gioia, finché non foste tornato: né....

\section{Castelloza:}

Non dovrei più desiderar cantare, poiché più canto peggio mi va l'amore, ché pianto e lamento han preso in me dimora; poiché in mala mercede ho riposto il mio cuore e me, e se in breve non me ne astengo, avrò aspettato troppo a lungo.

Ah bel amico, per lo meno mostratemi il vostro viso amabile prima ch'io muoia di dolore;

gli innamorati vi giudicano crudele, poiché da voi non mi proviene alcuna gioia; e tuttavia per questo non mi pento d'amar sinceramente ogni momento, senza volubilità di cuore.

Da quando vi vidi, fui in vostro potere, ed oltre a ciò, amico, non ebbi mai nessuno migliore di voi. Non inviatemi supplicante o messaggero a dirmi che oramai avete volto il freno altrove. Amico, non fate nulla: poiché gioia non mi sostiene, basta un nulla a farmi impazzire di dolore. 
Se ne avessi pro, ben vi ricorderei, cantando, che tenni il vostro guanto che rubai con gran timore; poi ebbi, poi ebbi paura che ne poteste aver danno da parte di colei che vi possiede, amico, per cui lo riportai indietro, dacché so bene di non averne diritto.

Anche il classico tema della lontananza, seppur raramente, trova posto nei versi delle trobairitz; qui è Clara d'Anduza che parla:

In grave angoscia e in grave tormento in grande follia hanno posto il mio cuore i maldicenti e i falsi indovini, che avviliscono joi e joven (gioia e gioventù) poiché hanno separato e allontanato da me voi che amo più di ogni cosa, cosicché non posso vedervi e ammirarvi per cui muoio di dolore, ira e rancore.

oppure Castelloza:

Siete stato a lungo lontano, amico, dacché partiste da me, ed è per me penoso e crudele, poiché mi giuraste e prometteste che per tutti i giorni della vostra vita non avreste amato altri che me; e se v'importa di qualcun'altra, allora mi avete uccisa e tradita, che io ponevo in voi la speranza che mi amaste senza incertezze.

\section{Relazioni tra donne}

La bellissima canzone di Bieiris di Roman, a mio parere la migliore tra tutte, è dedicata ad una signora Maria; si tratta di una poesia d'amore di una donna dedicata ad un'altra donna: 
Donna Maria, il pregio e la perfetta virtù, la gioia, il giudizio e la fine bellezza, l'umanità, il merito e l'onore, il parlar gentile e i graziosi modi, il dolce viso e la gaia leggiadria, il dolce sguardo e l'amabile aspetto che possedete e di cui non avete uguali mi attirano verso di voi con cuore sincero.

Per cui vi prego, se vi aggrada che amor cortese e gioia e dolce umiltà mi possano essere d'aiuto presso di voi, di donarmi ciò da cui spero d'aver gioia, poiché ripongo in voi il mio cuore e la mia volontà, e tutto ciò che mi rallegra proviene da voi, e a causa vostra sovente sospiro.

E poiché bellezza e virtù vi innalzano sopra ogni altra, e non v'è chi vi sopravanzi, vi prego, se vi aggrada, per ciò che vi fa onore, di non amare chi ha intenzione di ingannarvi.

Bella Dama, che pregio e gioia e parlar gentile innalzano, a voi mando le mie strofe, poiché da voi nasce la gioia e la felicità, ed ogni bene che si chiede ad una donna.

Anche nella già citata canzone di Azalais d'Altier circolano sentimenti, se non d'amore come in quella di Bieiris, certo di predilezione come testimoniano i versi che seguono:

E vi dico che in verità, non ho mai amato sinceramente una donna senza conoscerla; e vi dico anche, che Dio mi guardi, che al mondo non vi è alcuna cosa, che voi pensate vi piacerebbe, che io non farei volentieri, senza bisogno di ricompensa o di preghiera.

E, signora, ho troppo desiderio di vedervi... 
E' appena il caso di ricordare che il XII e il XIII secolo diventano così, per la grandissima influenza di queste donne (da non dimenticare nel nord sia Eloisa e Maria di Francia, sia Margherita Porete, Hadewich di Anversa, Beatrice di Nazareth, Giuliana di Norwich), i secoli dell'amore, periodo in cui si riscopre il Cantico dei Cantici, con le sue note d'esultanza e d'appassionato erotismo. Anche ad alcune lettere e poesie della severa Ildegarda di Bingen e alle risposte di alcune delle sue corrispondenti sono affidati messaggi di tenerezza amorosa. Certamente un contributo alla diffusione in Europa della civiltà cortese è stato dato da Eleonora d'Aquitania (1122-1204) che, personalmente e attraverso le figlie e i figli (Riccardo Cuor di Leone si cimentò con un certo successo nella poesia trobadorica), è presenza pervasiva e autorevole in Provenza prima e poi in Francia, in Inghilterra e in Spagna.

\section{Catarismo}

Forse questo sguardo sulla cultura occitanica medievale, sguardo volutamente rivolto in modo parziale, mancherebbe di un elemento significativo, se non accennassi al Catarismo, movimento religioso che si sviluppò soprattutto nella Francia meridionale, in Catalogna e in Piemonte, movimento che aggiunse all'identità culturale, linguistica e politica della Languedoc un'identità religiosa. Le donne nobili, ma non solo queste, furono molto presenti nel Catarismo, ricoprendo ruoli di responsabilità e prestigio, nella funzione di perfectae.

Il Catarismo, nelle sue forme più estreme, suggeriva l'astensione dai rapporti sessuali e dalla procreazione e forse la già citata tenso dibattuta tra Alais Iselda e Carenza verte su questo tema:

consigliatemi secondo la vostra esperienza: secondo il vostro parere, prenderò marito? oppure rimarrò pulzella ? Così mi piacerebbe, perché non credo sia bene fare figli;

Donna Carenza, fare bambini credo sia gran penitenza...

per cui vi consiglio, per far buona semenza, di prender per marito Coronato di Sapienza, 
da cui avrete il frutto di figli gloriosi;

chi lo sposa rimane pulzella.

La Chiesa condannò come eresia il Catarismo, ${ }^{8}$ scatenando una feroce repressione nella già ricordata crociata contro gli Albigesi (dal nome di Albi, noto centro cataro): troppo terrena, troppo femminile, troppo evoluta e indipendente appariva la Provenza! Esclarmonde di Foix, Giovanna di Tolosa, Giralda di Lavaur difesero fino allo stremo, dai loro castelli (la rocca di Monségur oppose una strenua resistenza), insieme al loro credo religioso, la civiltà cortese, con tutto quello che, al tempo e poi storicamente e letterariamente, ha significato per l'Europa. "Raffinata e colta" definisce questa cultura anche Germonda, nel suo sirventese, pur prendendone le distanze, perché partigiana della Chiesa di Roma.

Simone Weil, nel suo saggio L'ispirazione occitanica, ${ }^{9}$ parla della civiltà provenzale, che ammirava profondamente, come di "autentico Rinascimento", nel quale la concezione dell'amore, giunta alla sua pienezza, rifiuta la forza e compone armoniosamente i contrari, come solamente era avvenuto, secondo lei, nell'antica Grecia; per certi aspetti la Provenza fu centro dell'arte romanica, le cui proporzioni esprimono libertà ed equilibrio; la filosofa francese non si dà pace per la distruzione - che definisce assassinio - di questa civiltà e si strugge perché "E' infinitamente doloroso pensare che le armi di questo omicidio furono maneggiate dalla Chiesa. Altrove viceversa le sue riflessioni la inducono a esprimere parole di fiducia nella possibilità di rinascita di qualcosa di equivalente: "Ma la pietà custodita attraverso i secoli può consentire che un giorno nasca qualcosa di equivalente (...) Lo spirito della civiltà d'oc del XII secolo (...) corrisponde ad aspirazioni che non sono sparite e che non dobbiamo lasciar sparire, anche se non possiamo sperare di soddisfarle”.

\footnotetext{
${ }^{8}$ E. Bourassin. Les Cathares, Collection Charles Lavauzelle, Limoges-Paris, 1984; Monique Zerner-Chardavoine, La Croisade Albigeoise, Paris: Éditions Gallimard/ Julliard, 1979.

${ }^{9}$ Simone Weil, L'ispirazione occitanica, tr. G. Gaeta, in In forma diparole, Manuale secondo, ott.-dic. 1983, p. 90-112; L'agonia di una civiltà nelle immagini di un poema epico; ibidem, p. 88.
} 


\section{Corollario}

\section{I nomi delle trobairitz}

Le poesie note delle trobairitz sono $29 ;{ }^{10}$ le autrici (disposte qui in ordine cronologico quelle di cui si possiedono dati biografici o comunque notizie utili a situarle nel tempo) sono: Tibors, la Contessa di Dia, Almucs di Castelnau e Iseut di Capio, Azalais di Porcairagues, Maria di Ventadorn, Alamanda, Garsenda, Isabella, Lombarda, Castelloza, Clara d'Anduza, Azalais d'Altier, Bieiris di Romans, Gullelma di Rosers, Germonda di Montpellier; inoltre Alais, Iselda e Carenza, Donna H., Blanchemain, la Genovese, Bona Domna su cui non si ha nessuna notizia; ci sono poi sei poesie anonime. ${ }^{11}$ Dell'esistenza da Blanchemain ci parla Francesco da Barberino nel suo Documenti d'amore (da sistemare in nota: Francesco da Barberino, Documenti d'amore, a cura di F. Egidi, Roma, 1905-1927), non abbiamo di lei alcuna notizia diretta né tanto meno poesie; la Genovese non entra a pieno titolo nel novero delle trobairitz perché scrisse in dialetto genovese. La poesia "Amics en gran cossirier" è attribuita da J. Véran alla Contessa di Dia, mentre O. Schultz-Gora e Magda Bogin la considerano anonima; io ho seguito questi ultimi.

Probabilmente le trobairitz furono più numerose; A. Tavera ${ }^{12}$ scrive: "Su quattrocentosessanta trovatori, non ce n'è cinquanta di cui si conservino più di una dozzina di opere, e non ce n'è più di venticinque che lascino più di venti poesie; la norma, è il caso di Beatrice e di Castelloza, sono tre o quattro poesie... Io credo quindi che vi sia stato un maggior numero di trobairitz, che noi oggi non conosciamo, e che ciascuna abbia scritto più canzoni di quante ne siano sopravvissute". Al livello attuale degli studi, conosciamo, come è noto, una ventina, o poco più, di composizioni poetiche d'altrettante poetesse, "mentre un considerevole numero di altri testi è anonimo, testi per i quali sostengo valga il criterio della potenzialità"

${ }^{10}$ Per l'edizione completa delle poesie delle trobairitz il riferimento è ad Angelica Rieger, Trobairitz, Max Niemeyer Verlag Tubingen, 1991.

${ }^{11}$ Le Vidas e le Razos si trovano in J. Boutière e A. Schultz. Biographies des Troubadours, Paris: 1964; Marguerite Egan, Les vies des Trobadours, Bibliothèque Médiévale, Paris, 1985.

${ }^{12} \mathrm{~A}$. Tavera, "A la recherche des troubadours maudits", in Exclus et systèmes d'exclusions dans la litt. et la civil. médiévales, Paris:1978, p. 135-161. 
proposto da Angelica Rieger ${ }^{13}$ che dice ancora "dove non esistano prove contrarie e dove elementi di lingua e di contenuto lo consentano, essi possono essere ascritti ad autrici. Io ritengo che occorra abituarsi a declinare anche al femminile il termine anonimo". Saverio Guida scrive:

Quello dell'anonimato poetico è un terreno davvero minato, che richiede grande cautela da parte di chi vi si accosta o vi ci si avventura: troppo spesso si dimentica che "anonimo" non vuole dire affatto "di un uomo sconosciuto", può benissimo darsi che il testo adespoto sia opera di una donna. Io sono personalmente dell'avviso che una parte non trascurabile delle canzoni giunteci anonime vada ascritta a poetanti femminili e, per quanto estremistico e paradossale possa sembrare, non provo difficoltà a ipotizzare che alcuni dei testi circolanti sotto nome maschile abbiano in realtà come autori delle donne. ${ }^{14}$

Di moltissime Trovatore restano solo i nomi, Agnese, Caudairenga, Gullelma Monja, Blanceman, Lisa di Londres, Guiscarda, Huguette di Baulx, Lauretta di Sade, tra le altre l'opera risulta al momento smarrita, probabilmente (e già da più parti si ricerca in questa direzione) ad alcune delle poesie oggi anonime si potrà legare in futuro il loro nome. Della Contessa di Dia si conoscono quattro poesie, di Castelloza altrettante; di ciascuna delle altre ce ne resta una. Per Azalais di Porcairagues, Castelloza, la Contessa di Dia, Lombarda, Tibors abbiamo a disposizione la Vida; per Almucs, Maria di Ventadorn, abbiamo la Razo (la descrizione dei motivi che hanno dato origine alla poesia) che precede la poesia; per le altre le notizie si desumono dalla vida o dalla Razo di trovatori con cui furono in corrispondenza: per Alamanda la vida di Giraut di Bornelh, per la Bona Domna, la vida di Pistoleta, per Clara d'Anduza e Azalais d'Altier quella di Uc di SaintCirc. Per altre si possono fare congetture, raffronti, ipotesi. $^{15}$

\footnotetext{
${ }^{13}$ Angelica Rieger, in Prefazione, Le Trovatore, poetesse e poeti in conflitto, cit., p. 5-7.

${ }^{14}$ Saverio Guida, 'Trobairitz fantomatiche? I casi di Alamanda e di Escaronha", in Le rayonnement de la civilization occitane à l'aube d'un nouveau millanaire, a cura di G. Kremenitz, B. Czemlofsky, R. Tanzmeister, VI Congrès Internazionale de l'AIEO, 12-19 settembre 1999, Wien, 2001, p. 416.

${ }^{15}$ N. Viton de Saint-Allais, L'art de vérifier les dates, Paris: 1818.
} 


\section{Epigoni}

In seguito alcune mistiche, come Hadewijch di Anversa, ${ }^{16}$ troviere come la Duchesse de Lorraine,${ }^{17}$ riprenderanno temi e maniere delle poetesse cortesi così come altre, appartenenti allambiente stilnovistico italiano, di poco successive, come Compiuta Donzella, ${ }^{18}$ Nina Siciliana, con cui corrispose Dante da Maiano, Leonora di Genga, Livia da Chiavello, "le gentildonne di Fabriano" eccetera, ${ }^{19}$ le ebbero presenti e, nel loro amoroso cantare, ne continuarono la tradizione (io spero di riuscire a raccogliere notizie meno generiche sulle epigoni italiane). Le Preziose, qualche secolo dopo, legandosi strettamente a loro, ${ }^{20}$ ne ereditarono, rinnovandoli e attualizzandoli, l'interesse per la lingua, la cura per i rapporti, l'inclinazione per l'amore, energia civilizzatrice. ${ }^{21} \mathrm{Da}$

${ }^{16}$ G. Epiney-Burgard e E. Zum Brunn, Le poetesse di Dio. L'esperienza mistica femminile nel Medioevo, a cura di D. Bremer Buono, Milano: Mursia, 1984: lintroduzione di E. Zum Brunn, p.7-23. Hadijwich di Anversa, pp.108-124; brani delle opere di Hadijwich I e Hadijevich II, p.124-145; R. Guarnieri, Amiche mie, beghine, in AA.VV., Ruah. Il femminile di Dio, Roma: Stampa alternativa, 1997.

${ }^{17}$ M. Tyssens, AVoix des femmes dans la lyrique d'oïl, in AA. VV., Mélanges offerts à G. Dudy, Bruxelles: De Boeck Université, 1992, p. 374-387.

${ }^{18}$ L. Azzolina, La Compiuta Donzella di Firenze, Palermo, 1902; F. Flora, Storia della Letteratura Italiana, Milano: Mondatori, XI edizione, 1959, v. I, p. 41-42. ${ }^{19}$ Azzolina, La Compiuta, cit.

${ }^{20}$ Per i legami tra Trovatore e Preziose, vedi A. Guiducci, Il femminismo della camera blu e Madame de Lafayette, introduzione a La Principessa di Clèves, Milano: Rizzoli, 1986.

${ }^{21}$ B. Craveri, La civiltà della conversazione, Milano: Adelfhi, 2001; R. Lathuillère, La Préciosité. Étude historique et linguistique, Genève: Droz, 1969; A. M. Verna, Donne del Grand Siècle, Milano: Franco Angeli, 1994, Ch. Morlet-Chantelat, La Clélie de Mademoiselle de Scudéry. De l'épopée à la gazette: un discours feminin de la gloire, Paris: Honoré Champion, 1997; D. Denis, La muse galante. Poétique de la conversation dans loeuvre de M. de Scudery, Paris: Honoré Champion, 1997; C. Zamboni, Momenti radianti, in Diotima, Approfittare dell assenza. Punti di avvistamento sulla tradizione, Napoli: Liguori Editore, 2002, p. 171185. "La relazione" a firma di Marirì Martinengo e Marie Thérèse Giraud, "L'eredità delle Trovatore: dalle Trovatore alle Preziose", presentata all'VIII Congresso dell'AIEO di Bordeaux, 2005, sarà pubblicata sugli Atti congressuali 
indagare ancora la loro eco nelle poetesse italiane del '500, come Isabella Morra. ${ }^{22}$

\section{I tempi e i luoghi}

Le trobairitz vissero in un arco di tempo compreso tra il XII e il XIII secolo; Tibors, la prima conosciuta, nacque nel 1130, Germonda, che compose il suo sirventese tra il 1227 e il 1229, può essere considerata l'ultima, anche per il carattere della sua composizione, diversissimo dagli altri delle trobairitz.

I luoghi di origine o di residenza erano situati (e sono raffigurabili oggi) nelle zone della Francia limitate a sud dai Pirenei e dal Mediterraneo, a est dalle Alpi e dal Rodano, a ovest dall'Atlantico, a nord dalla Loira. Sono le regioni in cui fiorì la cultura trobadorica e dove si parlava la lingua d'oc, cultura cui le trobairitz appartennero, lingua in cui si espressero. Dopo la Crociata contro gli Albigesi non ci sono più tracce (al livello degli studi attuali) di trobairitz, a parte Germonda.

\section{Classe sociale d'appartenenza}

La quasi totalità delle trobairitz apparteneva all'aristocrazia: Tibors era sorella o figlia di Rimbaud d'Orange, la Contessa di Dia (forse si chiamava Beatrice) era probabilmente la moglie di Guglielmo di Poitier, Almucs (o Almodis) sposò Guiraut di Simiane, signore di Caseneuve, Apt e Gordes, Maria di Ventadorn era figlia di Elis di Castelnau e di Raimondo II, visconte di Turenne e sposò il visconte di Ventadorn; Garsenda era contessa di Provenza, Isabella forse figlia di Bonifacio del Monferrato o di Guido Marchesopulo, signore di Bodonitza in Tessaglia, e così via. ${ }^{23}$

Alcune trobairitz erano protettrici di trovatori, come Tibors, Maria di Ventadorn o Garsenda e incarnavano l'ideale della dompna; altre, numerose, erano amate o innamorate di trovatori: la Contessa di Dia e Azalais di Porcairagues amavano un Rimbaud d'Orange (nei secoli XI e XII i Rimbaud d'Orange sono stati tanti), Garsenda fu amata dai trovatori Gui di Cavaillon e Elias di Barjols; Lombarda fu amata da Bernatz N'Arnautz, Castelloza amò Arman di Brion, Gullelma fu amata da Lanfranco Cigala.

\footnotetext{
${ }^{22}$ I. Morra, Rime, Roma: Salerno Editrice, 2001.

${ }^{23}$ Cfr. Meg Bogin, The women troubadours, London-New York, 1976. p. 63-64.
} 


\section{Resumo}

Na Occitânia dos séculos XII e XIII havia uma sociedade feminina, onde as damas possuíam uma certa importância, e sua voz se fazia ouvir. As trobairitz são a expressão dessa sociedade. Num confronto entre a poesia occitana e a épica francesa observa-se bem a posição da dama. Nos partimens e nas tensos aparecem as mulheres como conselheiras e educadoras. Observe-se a estética e a ética na tenso de Gullelma di Rosers, o amor entre as damas em Bieiris di Romans e em Azalais d'Altier; a paixão em Castelloza, o tema do distanciamento em Clara d'Anduza e em Azalais di Porcairagues. A posição de relevo das mulheres, no movimento cátaro, assim como na língua e na cultura d'oc, foi importante na formação da identidade occitana. Segue um estudo que elenca os nomes das trobairitz, mesmo daquelas cuja obra se perdeu e que deixaram sua memória nos seus contemporâneos e nas gerações sucessivas; estudase aqui a questão do anonimato, do tempo e dos lugares em que floresceu a poesia occitana feminina, abordando a classe social das mulheres trovadoras, os pastiches, ou seja, algumas místicas e as stilnovistas italianas.

\section{Riassunto}

Nell'Occitania dei secoli XII e XIII c'era società femminile, cioè una società in cui si teneva gran conto delle donne e la voce femminile era ascoltata. Le Trobairitz sono espressione di tale società. Confronto fra la poesia occitana e l'epica franca riguardo alla posizione della donna. Nei partimen e nelle tenzoni appaiono in luce autorevole, ad esse uomini e donne ricorrono per consiglio; accettarono di svolgere funzione di educatrici; seguono esempi tratti dalle poesie. Confronto fra estetica ed etica nella tenzone di Gullelma di Rosers; amore fra donne in Bieiris di Romans e in Azalais d'Altier; la passione in Castelloza; il tema della lontananza in Clara d'Anduza e in Azalais di Porcairagues. Accenni al Catarismo e alla posizione di rilievo delle donne in questo movimento spirituale, che, insieme alla lingua e alla cultura, fu fautore dell'identità occitana. Segue un corollario che elenca i nomi di tutte le Trobairitz, anche di quelle la cui opera è andata perduta e/o che hanno lasciato memoria nei contemporanei e nelle generazioni successive; si affronta la questione dell'anonimato; si illustrano i tempi e i luoghi in cui fiorì la poesia occitana femminile, la classe di appartenenza delle poetesse, le epigoni delle Trobairitz, cioè alcune mistiche, le stilnoviste italiane. 